\title{
Plateau Modulus of Homogeneous Polystyrene/Poly(vinyl methyl ether) Blends
}

\author{
Shinichi Kitade, Kenji OchiaI, Megumi Ida, \\ Yoshiaki TAKAHASHI, and Ichiro NODA \\ Department of Applied Chemistry, Graduate School of Engineering, Nagoya University, \\ Nagoya 464-01, Japan
}

(Received March 24, 1997)

KEY WORDS Homogenious Blend / Polystyrene / Poly(vinyl methyl ether) / Zero-Shear Viscosity / Steady-State Compliance / Plateau Modulus / Scaling Theory /

Viscoelastic properties of miscible binary polymer blends depend on various factors such as the molecular weights and compositions of the components and the interactions between them.

In a previous work, ${ }^{1}$ we studied the zero-shear viscosity, $\eta^{0}$, and steady state compliance, $J_{\mathrm{e}}$, of homogeneous polystyrene/poly(vinyl methyl ether) (PS/PVME) blends with different molecular weights of PS, $M_{\mathrm{PS}}$, and a constant molecular weight of PVME, $M_{\mathrm{PVME}}$, maintaining the condition $M_{\mathrm{PS}} \gg M_{\mathrm{PVME}}$. $M_{\mathrm{PVME}}$ was $3.7 \times 10^{4}$ which is slightly larger than the molecular weight between entanglements, $M_{\mathrm{e}}\left(=1.2_{4} \times 10^{4}\right){ }^{2}$ Entanglements between PVME chains with such molecular weight are at most 1 or 2 per chain even in melts.

We reported that the PS volume fraction, $\phi_{\mathrm{PS}}$, dependence of zero-shear specific viscosity, $\eta_{\mathrm{sp}}^{0}$, at $\phi_{\mathrm{PS}}<$ 0.3 can be classified into dilute and semidilute regimes by critical $\phi_{\mathrm{PS}}$ values, $\phi_{\mathrm{PS}}^{*}$, and $\eta_{\mathrm{sp}}^{0}$ can be explained by the scaling theory ${ }^{3}$ in the semidilute regime where PS chains are entangled with each other, in the same way as in polymer solutions. ${ }^{4}$ Here, $\eta_{\mathrm{sp}}^{0}=\left(\eta^{0}-\phi_{\mathrm{PVME}} \eta_{\mathrm{PVME}}^{0}\right) /$ $\eta_{\mathrm{PVME}}^{0}, \eta_{\mathrm{PVME}}^{0}$ is the zero-shear viscosity of PVME and $\phi_{\mathrm{PVME}}=1-\phi_{\mathrm{PS}}$. In the same $\phi_{\mathrm{PS}}$ region, on the other hand, $J_{\mathrm{e}}$ exhibited the non-entangled regime behavior as those observed in corresponding polymer solutions, because the larger amounts of entanglements are needed to give the entangled regime behavior for $J_{\mathrm{e}}{ }^{5-7}$

In this work, we study the plateau modulus, $G_{\mathrm{N}}$, of PS/PVME blends to clarify the elastic property of the miscible blends in semidilute regime instead of $J_{\mathrm{e}}$. Here, we use a PVME sample with molecular weight lower than the previous sample, as well as the previous one to assure that the entanglements are composed of PS chains only.

\section{EXPERIMENTAL}

PVME samples used in this work were the previous one (fractionated one; $M_{w}=3.7 \times 10^{4}, M_{w} / M_{n}=1.32$ ) and a new one which was prepared by cationic polymerization in our laboratory following a literature, ${ }^{8}$ but using different terminator, $\mathrm{LiBH}_{4} \cdot{ }^{9}$ The number-average molecular weight, $M_{n}$, and the molecular weight distribution index, $M_{w} / M_{n}$, were determined to be $2.2 \times 10^{4}$ and 1.18 by ${ }^{1} \mathrm{H}$ NMR (unity-INOVA, Varian) and GPC (with Tosoh GMHXL mixed column $\times 2$ ), respectively. Hereafter, we call the previous one as PVME1 and the new one as PVME2. $M_{n}$ of PVME2 is about twice higher than $M_{\mathrm{e}}$ for PVME in melts, ${ }^{2}$ so that the entanglements of PVME chains are safely negligible in the blends with $\phi_{\mathrm{PS}}<0.3$. The PS samples were the same Tosoh standard polystyrenes with narrow molecular weight distributions, used in the previous work ${ }^{1} ;$ F-20 $\left(M_{w}=1.9 \times 10^{5}\right)$, F-40 $\left(M_{w}=3.6 \times 10^{5}\right), \quad$ F-80 $\quad\left(M_{w}=7.1 \times 10^{5}\right), \quad$ and F-126 $\left(M_{w}=1.26 \times 10^{6}\right)$. The preparation method of blend samples is the same as in the previous work. ${ }^{1}$

Rheological measurements were performed with a Mechanical Spectrometer RMS800 of Rheometrics Inc. using a parallel plate geometry with $2.5 \mathrm{~cm}$ diameter. Dynamic frequency sweep measurements were performed at several different temperatures $(323-373 \mathrm{~K})$ and all the data were shifted to the reference temperature $T_{\mathrm{r}}=353.6 \mathrm{~K}$ in accordance with the WLF equation for PVME. $^{2}$ This procedure was justified in the previous work, ${ }^{1}$ that is, the temperature dependence of shift factors, $a_{\mathrm{T}}$, of the blends agree with that of PVME. Dry nitrogen gas was introduced in the temperature control system of the RMS800 to prevent the oxidation of PVME and also the absorption of moisture.

\section{RESULTS AND DISCUSSION}

Examples of master curves of storage $\left(G^{\prime}\right)$ and loss $\left(G^{\prime \prime}\right)$ moduli of a PS/PVME2 blend at $353.6 \mathrm{~K}$ are shown in Figure 1. Some master curves of PS/PVME1 blends

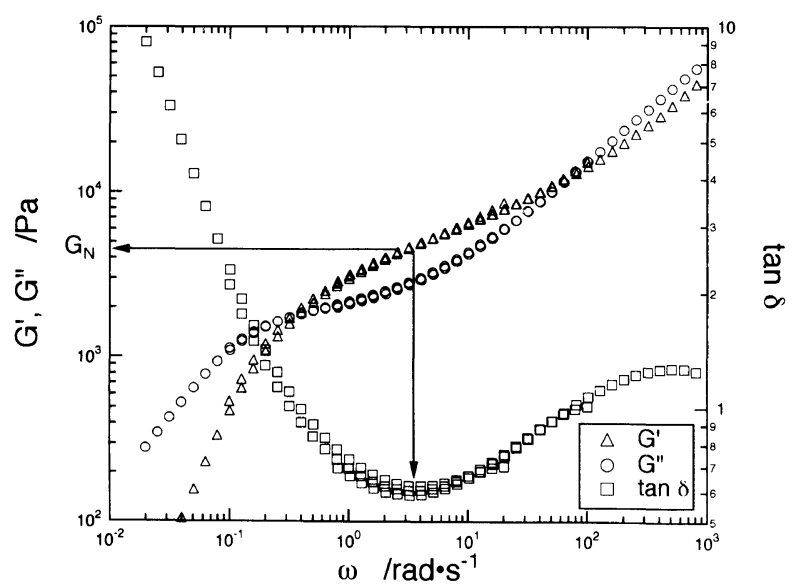

Figure 1. Example of the master curves of $G^{\prime}, G^{\prime \prime}$, and $\tan \delta$ at $353.6 \mathrm{~K}$ as a reference temperature. The sample is F-40/PVME2 blend with $\phi_{\mathrm{PS}}=0.244$. 

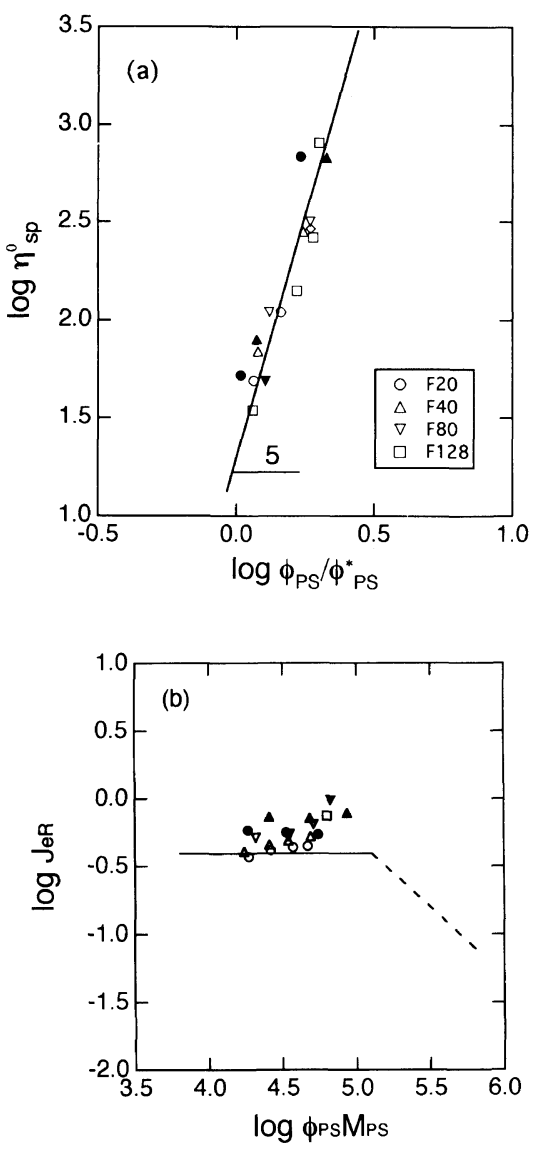

Figure 2. Double logarithmic plots of (a) $\eta_{\mathrm{sp}}^{0} v s . \phi_{\mathrm{PS}} / \phi_{\mathrm{PS}}^{*}$ for the blends and (b) $J_{\mathrm{eR}} v s$. $\phi_{\mathrm{PS}} M_{\mathrm{PS}}$. Different symbols denote difference of PS samples. The open and filled symbols denote the data of the blends with PVME1 and PVME2, respectively. Solid line in (a) denote the slope of scaling prediction, while solid and dashed lines in (b) denote the data of PS solutions in non-entangled and entangled regions, respectively. ${ }^{1}$

are already reported in the previous paper. ${ }^{1}$ Hereafter, all the rheological data will be discussed at this temperature. $\eta^{0}$ and $J_{\mathrm{e}}$ obtained in this work are consistent with the previous data ${ }^{1}$ as shown in Figures $2 \mathrm{a}$ and $\mathrm{b}$ in the form of $\eta_{\mathrm{sp}}^{0}$ vs. $\phi_{\mathrm{PS}} / \phi_{\mathrm{PS}}^{*}$ and reduced steady state compliance $J_{\mathrm{eR}}\left[=\left(J_{\mathrm{e}} \phi_{\mathrm{PS}} R T / M_{\mathrm{PS}}\right)\left\{\eta^{0} /\left(\eta^{0}-\eta_{\mathrm{PVME}}^{0}\right)\right\}^{2}\right] v s$. $\phi_{\mathrm{PS}} M_{\mathrm{PS}}$, respectively.

In the master curves of the blends with relatively low $\phi_{\mathrm{PS}}$ 's, the $G^{\prime}$ values are lower than the $G^{\prime \prime}$ values over all the $\omega$ range. On the other hand, in the master curves of the blends with relatively high $\phi_{\mathrm{PS}}$ 's $\left(M_{\mathrm{PS}} \phi_{\mathrm{PS}}>26000\right)$, the $G^{\prime}$ values become higher than the $G^{\prime \prime}$ values at the intermediate $\omega$ range, so that we can evaluate the plateau moduli, $G_{\mathrm{N}}$. Here, we determined $G_{\mathrm{N}}$ as the $G^{\prime}$ value at which $\tan \delta\left[=G^{\prime \prime} / G^{\prime}\right]$ value has a minimum as illustrated in Figure 1.

Figure 3 shows double logarithmic plots of $G_{\mathrm{N}}$ against $\phi_{\mathrm{PS}}$. The dashed line in Figure 3 represents the $G_{\mathrm{N}}$ data for PS solutions in $n$-benzyl buthyl phthalate ${ }^{10}$ (the reported concentration dependence is $\mathrm{C}^{2.2}$ to $\mathrm{C}^{2.3}$ ) plotted against polymer concentration instead of $\phi_{\mathrm{PS}}$. A similar concentration dependence $\left(\mathrm{C}^{2.4}\right)$ was also reported for PS solutions in Aroclor 1248. ${ }^{11}$ The $G_{\mathrm{N}}$ magnitudes of the blends and the solution are quite similar, confirming the previous conclusion ${ }^{1}$ that the entanglements are composed of PS chains only.

The experimental $\phi_{\mathrm{PS}}$ dependence of $G_{\mathrm{N}}$ determined

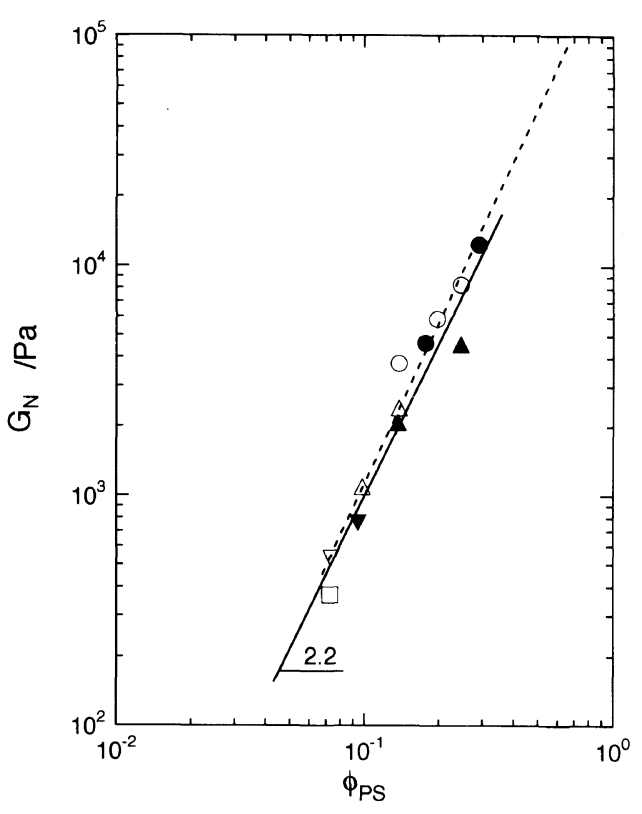

Figure 3. $\phi_{\mathrm{PS}}$ dependence of $G_{\mathrm{N}}$ for the blends at $\phi_{\mathrm{PS}}>\phi_{\mathrm{PS}}^{*}$. The data of PS in $n$-benzyl buthyl phthalate ${ }^{10}$ are represented by the dashed line with the slope of 2.2. Symbols are the same as in Figure 2.

by least squares method is given as

$$
G_{\mathrm{N}} \propto \phi_{\mathrm{PS}}^{2.2 \pm 0.1}
$$

If the scaling theory ${ }^{3}$ is applicable to $G_{\mathrm{N}}, G_{\mathrm{N}}$ is given by $G_{\mathrm{N}} \propto \phi_{\mathrm{PS}}^{3 v /(3 v-1)}$. Introducing $v=0.53$ used for the viscosity data as shown in Figure $2 \mathrm{a},{ }^{1}$ we have $G_{\mathrm{N}} \propto \phi_{\mathrm{PS}}^{2.7}$ for the PS/PVME blends, while we have $G_{\mathrm{N}} \propto \phi_{\mathrm{PS}}^{2}$ for the uniform network model. The experimental exponent is slightly larger than that of the uniform network model, but still much smaller than the value predicted by the scaling theory. As shown in Figure 3, the exponents of the blends and PS solution are practically the same. The exponents (2.2 to 2.3 ) for PS solution is nearly equal to the scaling prediction with $v=0.6$, but the data in semidilute region was smoothly connected with the data in highly concentrated region and melts by a straight line, just like the data of $J_{\mathrm{e}}{ }^{7}$ It was pointed out that the scaling theory need not to be brought in to understand concentration dependence of $J_{\mathrm{e}} \cdot{ }^{7}$ Likewise, the data for polymer blends can be smoothly extrapolated to melt data of PS, having an intermediate exponent of the scaling and uniform network model. Thus, we conclude that the $\phi_{\mathrm{PS}}$ dependence of the plateau modulus, i.e., the elastic property of homogeneous polymer blends is practically understood by the uniform network model similarly to polymer solutions. ${ }^{5-7}$

Acknowledgment. The authors thank to Prof. Sawamoto and Dr. Kamigaito of Kyoto University for helpful advise on the cationic polymerization of PVME. This work was supported by a Grant-in-Aid for Scientific Research (No. 08651074) from the Ministry of Education, Science, and Culture of Japan.

\section{REFERENCES AND NOTES}

1. S. Kitade, Y. Takahashi, and I. Noda, Macromolecules, 27, 7397 (1994).

2. Y. Takahashi, H. Suzuki, Y. Nakagawa, M. Yamaguchi, and I. 
Noda, Polym. J., 23, 1333 (1991).

3. P.-G. de Gennes, "Scaling Concepts in Polymer Physics," Cornell University Press, Ithaca, N. Y., 1979.

4. Y. Takahashi, Y. Isono, I. Noda, and M. Nagasawa, Macromolecules, 18, 1002 (1985).

5. J. D. Ferry, "Viscoelastic Properties of Polymers," Wiley, New York, N. Y., 1980.

6. W. W. Graessley, Adv. Polym. Sci., 16, 66 (1974).

7. Y. Takahashi, I. Noda, and M. Nagasawa, Macromolecules, 18,
2220 (1985)

8. M. Kamigaito, Y. Maeda, M. Sawamoto, and T. Higashimura, Macromolecules, 26, 1643 (1993).

9. This modification was proposed by Dr. Kamigaito and Prof. Sawamoto of Kyoto University.

10. Y. Isono, T. Fujimoto, N. Takeno, H. Kajiura, and M. Nagasawa, Macromolecules, 11, 888 (1978).

11 K. Osaki, Y. Nishimura, and M. Kurata, Macromolecules, 18, 1153 (1985). 